University for Business and Technology in Kosovo

UBT Knowledge Center

UBT International Conference

2015 UBT International Conference

Nov 7th, 9:00 AM - 5:00 PM

\title{
Microsimulation Models for Simulating Pathways of Patients with Mental Diseases
}

\author{
Andreas Bauer \\ Technische Universität Wien, andreas.e101.bauer@tuwien.ac.at \\ Felix Breitenecker \\ Technische Universität Wien, felix.breitenecker@tuwien.ac.at \\ Christoph Urach \\ dwh simulation service
}

Follow this and additional works at: https://knowledgecenter.ubt-uni.net/conference

Part of the Computer Sciences Commons, and the Digital Communications and Networking Commons

\section{Recommended Citation}

Bauer, Andreas; Breitenecker, Felix; and Urach, Christoph, "Microsimulation Models for Simulating Pathways of Patients with Mental Diseases" (2015). UBT International Conference. 108.

https://knowledgecenter.ubt-uni.net/conference/2015/all-events/108

This Event is brought to you for free and open access by the Publication and Journals at UBT Knowledge Center. It has been accepted for inclusion in UBT International Conference by an authorized administrator of UBT Knowledge Center. For more information, please contact knowledge.center@ubt-uni.net. 


\title{
Microsimulation Models for Simulating Pathways of Patients with Mental Diseases
}

\author{
Andreas Bauer ${ }^{1}$, Felix Breitenecker ${ }^{1}$, Christoph Urach ${ }^{2}$ \\ 1 TU Wien, Institute for Analysis and Scientific Computing, Wiedner Hauptstraße 8- \\ 10, Vienna, Austria. \\ 2 dwh simulation services, Neustiftgasse 57-59, Vienna, Austria. \\ andreas.e101.bauer@tuwien.ac.at
}

\begin{abstract}
Predicting demand for care is necessary to provide sufficient capacities in hospitals and enable better planning to cope with challenges like changes in the structure of the population. Emphasis in this work is put on the analysis of the data and on the parametrization of a simulation model for the paths of patients with mental diseases through the health care system. Survival analysis and model selection methods are used for this purpose. Data on patients and their treatments is analyzed with methods of survival analysis. Different methods for modelling the survival and hazard function are presented and compared. Hereby, the focus is on the cox model. It is used to model the hazard function and can be extended to analyze multiple events. With the use of model selection methods the significant parameters are determined. Only these are included in the simulation model. These methods shall help to raise the quality of the parametrization and therefore the whole simulation model. In the microsimulation model, every patient has a particular set of parameters and can be in one of several predefined, exclusive states. The events are implemented as state changes. The probabilities for the events are calculated using the hazard functions. These are estimated with several extensions of the cox model. The considered events in the simulation are readmissions to hospital and contacts to ambulant psychiatrists. The simulation runs for a predefined time span and the sequence of events of each patient is tracked. After the simulation, the individual paths of the patients as well as aggregated quantities such as the overall numbers of certain events are analyzed. Simulations for different populations are performed. The results for various scenarios are presented. Scenarios with and without contacts to a psychiatrist are considered as well as different maximum numbers of admissions. Also, the subpopulations are compared. For example, differences in the results for diagnosis groups are encountered. These simulations shall lead to an improvement of the prediction of the pathways of the patients and therefore help to evaluate interventions like treatment changes the health care system or the utilization of the capacities in hospitals.
\end{abstract}

Keywords: Microsimulation, Survival analysis, Cox regression

\section{Introduction}

Predicting demand for care is necessary to provide sufficient capacities in hospitals and enable better planning to cope with challenges like changes in the structure of the population. This work deals particularly with patients with mental diseases. For the purpose of simulating the demand of these patients, a microsimulation model is built. In order to improve the quality of these simulations the emphasis in this work is put on the analysis of the data and on the parametrization of the simulation model for the pathways of patients with mental diseases through the health care system. Survival analysis methods are used for this purpose. The focus is put on the Cox model and its extensions.

\section{Methods}

Methods from the field of survival analysis are used to build the statistical model behind the simulation model. Survival analysis deals with the analysis of data of the time until the occurrence of 
a particular event. This kind of data is frequently encountered in medical research and also in other areas of application and referred as survival data. In this work the considered event are readmission to hospital, contact to a psychiatrist and death. The methods allow the estimation and the analysis of the survival function and the hazard function. The survival function is defined as the probability that an individual will survive up to time and the hazard function is defined as the instantaneous rate of death at time. The cumulative hazard function is given by. The Nelson-Aalen estimate is an estimate for the cumulative hazard function. Let and denote the numbers of people that experience the event at time $t$ respectively are at risk at time t. Let denote the event times. Then, can be estimated by

The Cox model is a model for the hazard function [1]. It assumes that the ratio of the hazards of different exposure groups remains constant over time. This is called the proportional hazards assumption. The mathematical form of the model is:

The baseline hazard refers to a particular group of individuals (for example, the individuals with value zero in all categories), is the number of covariates, is the value of the th covariate and is the corresponding regression coefficients.

An extension of the Cox model is the stratified Cox model [2]. This model allows multiple strata. The strata divide the subjects into disjoint groups and each subject is member of exactly one stratum. Each of which has a distinct baseline hazard function but common values for the coefficient vector. The overall $\log$ likelihood is the sum of the log likelihoods of each stratum. In the multi-state model every transition between states is possible [2]. Every transition defines a stratum. So, the stratified Cox model can be applied. The transition probabilities can be calculated from the cumulative transition hazards [3]. Let be a time-inhomogeneous Markov process with state space. The cumulative transition hazards, can be estimated through the multi-state extension of the Cox model and. This leads to the approximation of the transition probabilities with a partition of the interval $[\mathrm{s}, \mathrm{t}]$ and:

\section{Model}

The chosen model type is a microsimulation model. Iit follows the bottom-up approach and every single individual is modelled. This approach is chosen because not only the cross-sectional analysis is important but also the longitudinal pathways of individuals. This approach is suitable for analysis of different policies and scenarios. The characteristics of the individuals are manageable with a bottom-up approach.

In the microsimulation model, every patient has a particular set of parameters and can be in one of several predefined, exclusive states. Patient parameters are sex, age, length of stay in the psychiatric department of the hospital and diagnosis. The events are implemented as state changes. The possible ways through the states are described by a transition matrix which can be interpreted as a directed acyclic graph. The transitions are evaluated using first-order Monte Carlo simulation by comparing a random number with the given transition probability. The probabilities for the events are calculated using the hazard functions for the transitions. These are estimated with the multi-state model. The considered events in the simulation are readmissions to hospital, contacts to ambulant psychiatrists and death. The simulation runs for a predefined time span and the sequence of events of each patient is tracked. Every individual starts in state R (released after the first admission to hospital). If the most recent event of the patient was the i-th readmission, it is in state, if the most recent event was the $\mathrm{i}$-th ambulant psychiatrist visit, the patient is in state and if the patient died, it is in state. From initial state transitions are possible to the states, and. From the patient can go to and from to, and to. From death no transitions are possible since it is an absorbing state.

\section{Simulations}


Various simulations of the microsimulation model are carried out for a simulation time of two years. Stacked area plots of the evolutions of the patient's distribution over the states comparing psychotic and nonpsychotic patients are presented in Figure 1.
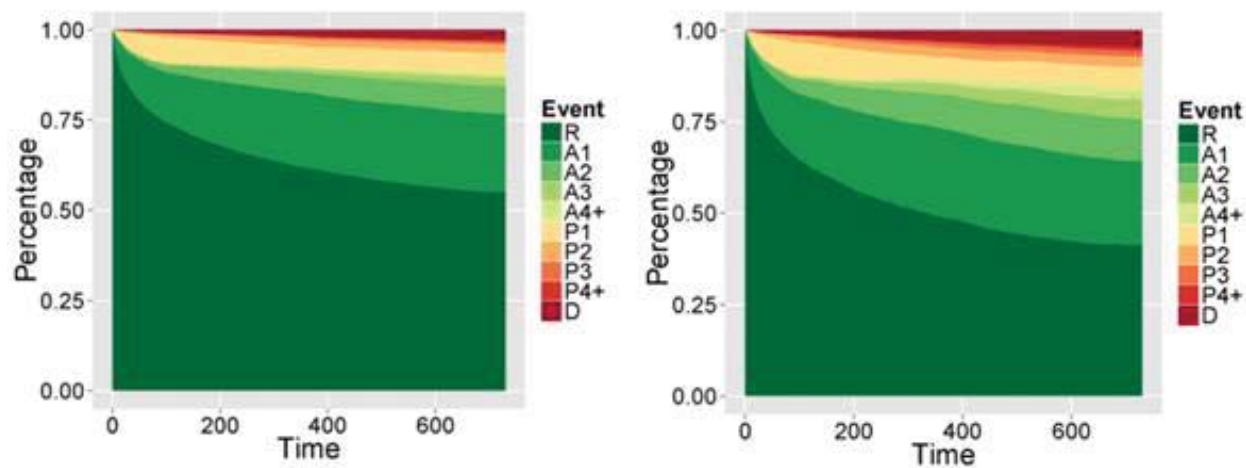

Fig. 1. Evolution of the patients distribution over the states for psychotic and nonpsychotic patients

The percentage of psychotic patients still remaining in state is decreasing much faster than for nonpsychotic. At the end of the simulation $41 \%$ of the psychotic patients are still in state while about $55 \%$ of the non-psychotic patients are in state. The percentages of patients in the psychiatrist states run very similarly in both subpopulations.

\section{Summary and Outlook}

A microsimulation model for the simulation of the pathways of patients with mental diseases was successfully implemented. The Cox model proved to be a helpful tool for the statistical model. The main benefits of the Cox model are the inclusion of patient parameters and the flexibility for extensions for multiple events. The first simulation results already outline a difference between psychotic and nonpsychotic patients in terms of the number of events. Psychotic patients have significantly more events during the simulation than nonpsychotic ones.

Work that is still open is a deeper analysis of the simulation results. Also, the definition and comparison of different scenario will be executed and the usefulness of an intervention strategy will be tested.

\section{References}

1. Cox, D. R.: Regression models and life-tables. Journal of the Royal Statistical Society. Series B (Methodological) (1972) 187-220

2. Therneau, T. M., Grambsch, P. M.: Modeling survival data: extending the Cox model. Springer Science \& Business Media, 2000.

3. J. Beyersmann, J., Putter, H.: A note on computing average state occupation times.

Demographic Research (2014), vol. 30, no. 62, 1681-1696 